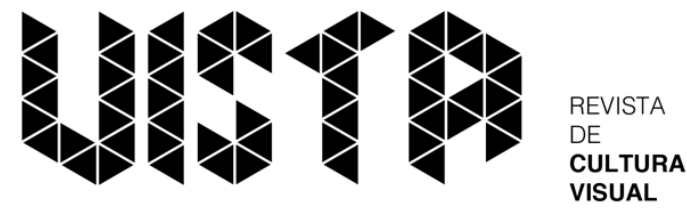

\section{Sobre as visualidades urbanas}

\section{Ricardo Campos, Andrea Barbosa \& Cornelia Eckert}

A cidade é um universo que pode ser concebido a partir de diversos pontos de vista. É, desde logo, um território, com fronteiras formalmente definidas e com uma geografia que é ocupada por diferentes materialidades e seres (humanos e não-humanos). É, também, um universo social, na medida em que é habitada e vivida, ao longo do tempo, por diferentes pessoas e grupos sociais que vão produzindo a cidade. O espaço e o tempo são, assim, componentes da cidade que é fabricada em camadas, marcada pela permanente mudança da sua paisagem. Mas a cidade também é imaginário, é construída mentalmente não correspondendo necessariamente às suas configurações físicas concretas. Deste modo, a cidade imaginária é aquela que habita as nossas mentes mas, também, cada vez mais, aquela que se fabrica através dos circuitos digitais e dos ecrãs, com distintas conexões à realidade concreta que habitamos.

Estes são patamares diversos onde a cidade se pensa e se constrói enquanto realidade vivida por diferentes gerações, que se sucedem na trama de complexas experiências. A questão da visualidade merece ser pensada a partir destes múltiplos pontos de vista, procurando entender como a cidade se fabrica, mas também como é comunicada e entendida (Campos, Brighenti \& Spinnelli, 2011; Barbosa, 2012). Não falamos de abstrações, mas situações muito concretas através das quais nós erigimos edifícios, viadutos, ruas e parques, uma extensa malha de equipamentos urbanos em que a vida quotidiana é praticada. Espaços vividos através oas quais comunicamos, recorrendo a cartazes, ecrãs, graffiti, pixação, vitrines, sinalizações, etc. A visualidade é parte central deste contexto. Ela demarca e agencia diferentes cidades vividas, cidades sonhadas e cidades negadas (Barbosa, 2012). A visualidade tem uma componente funcional, mas igualmente política, estética e simbólica. Serve para ordenarmos o mundo e a sua paisagem, de acordo com modelos dominantes, que não deixam de ser desafiados. A visualidade remete para a forma como social e historicamente o olhar (ferramenta de 
perceção) e o visível (percecionável) são construídos (Campos, 2013). Logo, trabalhar sobre as visualidades urbanas implica adotar este múltiplo sentido, analisando a forma como olhamos a cidade, mas também como esta se dá a ver. Mas implica também, pensar processos e atores sociais. Quem intervém no campo da visualidade, de que modo e com que intuito, são interrogações fulcrais. As questões do poder e da agência são, por isso, trazidas a debate, demonstrando que as relações no campo da visualidade são assimétricas. Logo, falar de visualidade é, também, pensar o domínio da invisibilidade (Campos, 2016), questionando porque certas pessoas, grupos ou ações permanecem na penumbra ou fora da vista.

Esta é uma matéria ainda mais complexa quanto, como vimos, a cidade não existe apenas no campo da materialidade, mas cada vez mais também num patamar chamado de virtual, em constante articulação com o mundo físico. Entre tantos outros, instrumentos como o Google street view permitem-nos navegar pelas cidades sem sair do nosso sofá. Mas nós também somos agentes ativos nesse processo. As selfies, os vídeos distribuídos através do youtube, entre muitos outros recursos e plataformas, são fragmentos da cidade e do nosso quotidiano. Ao colocarmos nas plataformas digitais imagens da nossa presença física na(s) cidade(s) estamos a criar meta-narrativas imagéticas sobre a cidade, que se reproduzem velozmente no ciberespaço, dando origem a outros cruzamentos de significado. A cidade é, por isso, também feita de uploads, shares e likes, numa nova gramática global.

Mas porquê centrar-nos na questão da visualidade? Não podemos ignorar que ver é algo inerente à forma como nos situamos na realidade concreta. Ver serve para nos orientarmos no mundo, mas também para comunicarmos com os outros e com tudo aquilo que nos rodeia. Tal como afirmava John Berger numa frase celebrizada: "a vista chega antes das palavras. A criança olha e vê antes de falar [...]. A vista é aquilo que estabelece o nosso lugar no mundo que nos rodeia." (Berger, 1999: 11).

Podemos falar de uma condição urbana em que a visão e a imagem são protagonistas de destaque? A sua importância parece-nos evidente. Em primeiro lugar, porque, como diversos autores vêm apontando, nós vivemos numa sociedade ocularcêntrica (Synnott, 1992; Jencks, 1995; Classen, 1997). O que esta afirmação significa, de modo mais simplificado, é que a nossa sociedade tem vindo a privilegiar ao longo da história a visão como o mais poderoso dos sentidos humanos. Ou seja, apesar de a nossa experiência no mundo ser de natureza multissensorial, a cultura ocidental e urbana tem vindo lentamente a aperfeiçoar os mecanismos de perceção visual do mundo, em detrimento dos outros sentidos. Em segundo lugar e em estreita conexão com esta condição, está o facto de vivermos numa era onde a tecnologia tem um papel fundamental no nosso dia-a-dia. Se a tecnologização da sociedade é uma evidência, não podemos esquecer 
a centralidade que a maquinaria ótica e visual assume. Mirzoeff afirma, por isso, que "a vida moderna desenrola-se no ecrã (...) a experiência humana é atualmente mais visual e visualizada do que alguma vez antes" (Mirzoeff, 1999, p.1). Nesta cultura altamente tecnológica, não somos apenas consumidores de media e de imagens, mas também criadores de conteúdos imagéticos e mediáticos (Burgess e Green, 2009). Como nos diz Muller (2008: 102), a produção (áudio)visual "privatizou-se enquanto a disseminação se globalizou". Isto significa que o cidadão comum dispõe hoje de um conjunto de competências e tem acesso a um rol vasto de ferramentas que permitem uma maior produção e circulação de bens visuais. A cidade é, como afirmámos, frequentemente palco ou suporte para a produção de imagens contemporâneas.

A visualidade na cidade também nos endereça para questões de ordem política. Quem age na cidade compondo a sua paisagem, que intuitos e estratégias utiliza? As questões de poder têm sido debatidas no âmbito da produção e difusão de imagens, mas também na esfera do olhar e das relações assimétricas que o compõem. Os atores mais poderosos produzem paisagem, regulam-na e vigiam-na, impondo uma ordem visual e uma "geografia moral" (MacAuliffe, 2012) sobre a cidade que, apesar de naturalizada, é constantemente desafiada por um conjunto de múltiplos agentes. Como tal, a cidade também é palco de movimentos disruptivos, catalogados imediatamente pelos poderes e pela moral dominantes como perigosos, anómalos ou ilícitos.

Esta importante dimensão da vida humana, durante demasiado tempo desvalorizada pelas ciências sociais e humanas, tem atualmente despertado a atenção de investigadores de distintas áreas académicas. Hoje encontramos diversas pesquisas que se focam sobre as imagens e a experiência visual urbana, sobre a forma como produzimos e consumimos imagens. Neste âmbito o espectro analítico e metodológico tem vindo a alargar-se. Recorrendo à etnografia visual, ao uso da fotografia, do vídeo, à ilustração ou hipermédia, vários são os procedimentos utilizados, que trabalham em aliança com as técnicas e metodologias mais convencionais.

Os organizadores do dossiê assumem esta forte ligação à imagem, quer enquanto objeto de estudo, quer enquanto dispositivo epistemológico, no campo das ciências sociais. Neste contexto é de destacar a relevância da antropologia visual, um campo específico da disciplina antropológica que, há décadas, vem promovendo um forte debate em torno destas questões (Eckert \& Monte-Mór, 1999; Barbosa \& Cunha, 2006; Barbosa, Cunha \& Hikiji, 2009; Barbosa, Cunha, Hikiji \& Novaes, 2016). É significativo o patrimônio etnográfico que a antropologia brasileira e a antropologia portuguesa hoje apresentam para a comunidade internacional, tendo por base a produção audiovisual em contextos urbanos. Não apenas como instrumentos de coleta de dados, mas em 
especial como referentes epistemológicos na reflexão sobre o lugar da imagem e da imaginação nas múltiplas formas de redes e de interações sociais.

Motivados em divulgar estas experiências de pesquisa que tematizam as visualidades urbanas, este dossiê testemunha uma profícua troca intelectual luso-brasileira. Daí que seja de destacar a forte presença neste dossiê de contributos de autores provenientes de instituições académicas brasileiras, revelando a intensa produção que se vem desenvolvendo neste país, ao nível das temáticas relacionadas com a imagem e as tecnologias visuais na pesquisa social. Neste volume trazemos contributos que configuram este movimento contemporâneo. Ora refletindo sobre o campo imagético no viver urbano, ora considerando a reverberação das tecnologias digitais nas práticas quotidianas.

Com este dossiê da Vista - revista de cultura visual - procurámos explorar esta dimensão específica da experiência urbana. Reunimos um total de nove artigos e dois ensaios visuais que nos trazem uma grande diversidade de ângulos e de pesquisas revelando que, de facto, este é um campo complexo.

O dossiê abre com um artigo que propõe discutir o espaço público urbano como um território para o exercício da ação política. Kando Fukushima e Marilda Queluz, no seu artigo "Produção do espaço e resistência: uma reflexão sobre cartazes de contestação", trazem a debate as apropriações dos espaços públicos urbanos em duas cidades brasileiras (Curitiba-PR e São Paulo-SP), para a distribuição de cartazes de contestação política. A partir de uma pesquisa realizada pelos autores, é evidenciado o carácter informal, inusitado e contemporâneo destas formas de expressão que revelam resistência e criatividade no uso da rua e dos seus dispositivos. Aquilo que os autores nos demonstram é que a participação política também se realiza nestes contextos, sendo que a imagem e a comunicação visual na rua podem assumir um papel muito relevante nos variados combates ideológicos em curso.

No artigo "L'expérience de la flânerie dans Les mains négatives (1979), de Marguerite Duras" a autora Luciene Guimarães de Oliveira nos convida a rever ou conhecer a curta metragem citada no titulo. O espectador é seduzido para uma flânerie na cidade de Paris guiada pela poética durassiana. A cidade moderna se revela em suas passagens e fragmentos pela arte do cinema emergente, produto técnico desta modernidade. A poética de Duras, recitada em off, produz um arranjo complexo da estética citadina pela câmera em travelling, testemunha de uma nova rítmica temporal descontinua. A narração assimétrica, fragmentada, favorece a abertura do significado e assume, segundo Duras, o papel do livro, espaço que deve ser preenchido pela imaginação do leitor, para ver mais do que é representado na tela, orienta a autora. 
"Ningún pibe nace chorro" de Andrea Molfetta traz uma breve etnografia dos atores, práticas e processos do Centro de Producción Audivisual de la Casona de Florencio Varela, situado na região metropolitana de Buenos Aires, Argentina. A autora analisa o discurso contruído pelos jovens nas suas curta-metragens sobre as representações que a televisão difunde sobre as suas vidas enquanto habitantes da periferia de Buenos Aires. O argumento da autora é de que as práticas enunciativas do cinema comunitário realizado por esses jovens é uma resposta ao discurso hegemónico que os estigmatiza. O discurso agenciado nesses filmes é reflexivo, meta-mediático e trans-midia no seu sentido político, porque cria a possibilidade de uma visualidade comunitária própria, na qual os jovens vinculam-se para construir e sonhar saídas para tudo aquilo de que não gostam em suas vidas quotidianas. No final a autora realiza uma breve análise de duas curta-metragens produzidos neste contexto numa narrativa que enfrenta a narrativa televisiva o que possibilita abrir outras possibilidades para a estigmatização, afinal "Ningún pibe nace chorro" (nenhuma criança nasce ladrão).

O crescimento exponencial da produção fotográfica vem gerando forte impacto na veiculação da imagem cultural das cidades contemporâneas, especialmente quando colocamos em discussão o género fotográfico selfie. "Fotografia Selfie em festivais: Experiência cultural como dispositivo para a nova imagem urbana" de Paulo Nunes, propõe uma discussão sobre a revitalização da imagem urbana pela cultura e o papel ocupado pela fotografia neste processo. Os usos sociais da imagem fotográfica, tão comuns no quotidiano das redes sociais, têm deslocado a produção da identidade da cidade para o âmbito da experiência. A partir da interação entre público e lugar (fotografia e vídeo), criam-se espaços físicos simulados, interferindo na estrutura das cidades em larga medida pela ordem imagética. $\mathrm{O}$ argumento de que o dispositivo fotografia-memória-experiência, accionado durante as diversas práticas de consumo cultural urbano, modifica o modo como o sujeito vivencia a cidade e fornece uma retórica imaterial que a reconstrói, ressignificando sua imagem a cada share ou like conquistado nos canais virtuais.

Afirmámos que a cidade contemporânea não existe apenas enquanto território e materialidade. Cada vez mais ganha existência em écrans e circuitos desmaterializados, numa realidade híbrida que viaja entre o espaço "online-virtual" e o "offline-material". O texto de Daniel Abath, intitulado "Visualidades sociotécnicas da cidade no jogo eletrônico Watch_Dogs", visa precisamente discutir a influência dos jogos eletrônicos na construção sociotécnica das representações de cidades - em específico a cidade de Chicago. A proposta é a de refletir sobre os mecanismos geradores de uma experiência urbana virtual a partir de observações das cidades físicas. Aquilo que o autor demonstra é que as visualidades urbanas são delineadas e consumidas também em diferentes 
plataformas tecnológicas e digitais, deste modo reinventando as nossas cidades e a nossa experiência urbana.

O artigo de Andreas Valentin, intitulado "Berlin<>Rio: trajetos e memórias", tem por motivação a memória familiar restabelecida numa narrativa de imagens. A partir de uma estadia de estudo em Berlim, o autor retoma material fotográfico produzido por seu pai. Percorre os mesmos trajetos em busca de pistas, buscando desvendar, como diz o autor, camadas de memória que, direta ou indiretamente, dialogassem com sua ancestralidade. Contrapõe as fotografias tiradas por seu pai, com as suas, produzidas nesta revisitação aos lugares de memória. A história familiar relatada e vivida entre Berlin e Rio de Janeiro, para onde seus familiares migraram, revela um movimento migratório muito presente nas memórias familiares de brasileiros. O retorno a Berlin é também um projeto de pesquisa e de produção artísticas, exposta este ano no centro cultural Haus am Kleistpark, Berlin (exposição “Berlin<>Rio: Spuren und Erinnerungen”). A reminiscência, neste evento cultural, é testemunhada com outros documentos e a linhagem geracional se alonga na ascendência. $O$ autor apresenta estas imagens e orienta o leitor sobre a produção técnica do material exposto.

Lindolfo Sancho no seu artigo "Antropologia no campo expandido: uma articulação entre espaço, paisagem e as artes no estudo da experiência urbana" parte da ideia de que fora dos museus e em interação com o espaço público, as artes se tornam objeto privilegiado de mediação da experiência urbana. Tendo essa experiência no horizonte, o artigo apresenta uma reflexão sobre duas intervenções realizadas por Gordon MattaClark nos pieres de Nova lorque durante a década de 1970. Considerando as categorias de espaço e paisagem como ferramentas teóricas, a mobilização do espaço urbano pelas artes é considerada a partir da ideia de "campo expandido", tal como proposto por Rosalind Krauss referindo-se a um momento histórico no qual as fronteiras entre as práticas artísticas foram flexibilizadas em uma série de práticas espaciais híbridas. A triangulação entre espaço, paisagem e arte, que trazem a experiência urbana como objeto de reflexão é abordada a partir da ideia de paisagem enquanto legado das artes a operar como mediador de uma experiência visual no espaço urbano.

Os dois contributos que encerram a secção de artigos deste dossiê remetem para uma temática que tem sido crescentemente abordada pela literatura em ciências sociais, nomeadamente no campo dos estudos urbanos. Referimo-nos ao graffiti e à arte urbana, elementos indissociáveis da paisagem urbana contemporânea. Jose Luis Junior, com o artigo intitulado "Quem sujou as mãos de tinta? Estética, gesto e matéria em intervenções artísticas urbanas", leva-nos a pensar sobre as intervenções artísticas de rua na cidade de Porto Alegre, no sul do Brasil. Acompanhando um conjunto de artistas urbanos nesta cidade, a pesquisa etnográfica levada a cabo pelo autor permite-nos olhar 
de forma original para estes agentes criativos e para o impacto que as suas obras têm na paisagem urbana. A cidade construída é, também, o resultado destes gestos mais ou menos informais, ou legais, realizados por artistas que pertencem a uma comunidade com fronteiras identitárias e simbólicas definidas.

Por seu turno, numa abordagem bem distinta, Rafael de Freitas, conduz-nos à maior metrópole da América do Sul, São Paulo. Através do seu artigo "Intervenções gráficas no espaço público urbano: uma abordagem antropológica da cidade de São Paulo", o autor propõe-nos uma deambulação em torno de diferentes circuitos e paisagens urbanas, em diálogo com as múltiplas intervenções gráficas encontradas no espaço público. Graffiti e pixação são, entre outros, intervenções omnipresentes, confundindose com outras tantas matérias visíveis no edificado. Estas inscrições desvendam circuitos de mobilidade urbana e de pertença grupal, são formas de comunicação codificada que utilizam os recursos disponíveis na cidade para se tornarem visíveis e adquirirem vida. É impossível pensar a cidade de São Paulo sem a sua presença.

Ainda como parte do dossiê, apresentamos dois ensaios visuais. "Escenas de Cambuci" de Vivian Castro é parte de um projeto pessoal da autora ainda em curso sobre o bairro de Cambuci, São Paulo. Conhecido por ser um bairro histórico da cidade, Vivian oferece-nos uma narrativa livre a partir da sua própria experiência como moradora. Cenas quotidianas são observadas e registadas por meio de anotações textuais e fotografias realizadas com uma câmara analógica.

A segunda narrativa visual que trazemos resulta da experiência de pesquisa etnográfica desenvolvida pela equipe do Núcleo de Antropologia Visual do Programa de PósGraduação em Antropologia Social da Universidade Federal do Rio Grande do Sul, Brasil. O exercício etnográfico coletivo realizado de abril a junho de 2017 , abordou o tema da arte urbana na cidade de Porto Alegre (RS). O trabalho interpretativo das imagens produzidas foi orientado pela leitura e aprendizagem conceptual de obras de nove intelectuais com estudos sobre cidade e grupos urbanos. O material foi exposto de novembro de 2017 a janeiro de 2018 na Reitoria da UFRGS com o lançamento de um catálogo da exposição.

\section{Referências Bibliográficas}

Barbosa, A. (2012) São Paulo Cidade Azul. São Paulo: Alameda.

Barbosa, A. \& Cunha, E. (2006), Antropologia e Imagem. Rio de Janeiro: Jorge Zahar Editor.

Barbosa, A., Cunha, E. \& Hikiji, R. (2009). Imagem-Conhecimento. Antropologia, Cinema e Outros Diálogos. Campinas: Papirus. 
Barbosa, A.; Cunha, E.; Hikiji, R. \& Caiuby Novaes, S. (2016) A Experiência da Imagem na etnografia. São Paulo: Terceiro Nome.

Berger, J. (1999) Modos de ver. Lisboa: Edições 70

Burgess, J. \& Green, J. (2009). Youtube: online video and participatory culture. Cambridge: Polity Press

Campos, R. (2016) Visibilidades e invisibilidades urbanas, Revista de Ciências Sociais, Vol 47, $n^{\circ} 1$ : 49-76.

Campos, R. (2013) Introdução à cultura visual. Abordagens e metodologias em ciências sociais. Lisboa: Mundos Sociais.

Campos, R; Brighenti, A. \& Spinelli, L. (Org.) (2011) Uma cidade de Imagens. Produção e consumo visual em meio urbano. Lisboa: Mundos Sociais.

Classen, C. (1997). Fundamentos de una antropología de los sentidos. Revista Internacional de Ciencias Sociales (RICS), $n .^{\circ} 153$.

Eckert, C. \& Monte-mor, P. (orgs.) (1999). Imagem em Foco. Novas Perspectivas em Antropologia. Porto Alegre: Universidade Federal de Rio Grande do Sul.

Jenks, C. (1995) Visual Culture, Londres e Nova lorque: Routledge.

Mcauliffe, C. (2012) Graffiti or street art? Negotiating the moral geographies of the creative city. Journal of urban affairs, 34 (2), 189-206.

Mirzoeff, N. (1999) An introduction to visual culture, Londres e Nova lorque: Routledge.

Muller, M. (2008). Visual competence: a new paradigm for studying visuals in the social sciences. Visual Studies, XXIII (2), 101-102.

Synnott, A. (1992) The eye and I: a sociology of sight. International Journal of Politics, Culture and Society, Vol 5, $\mathrm{n}^{\circ} 4,617-636$.

Ricardo Campos é Doutorado em Antropologia Visual. É investigador integrado (Investigador FCT) no CICS.Nova (Centro Interdisciplinar de Ciências Sociais da Faculdade de Ciências Sociais e Humanas) e professor convidado no Mestrado em Relações Interculturais (Universidade Aberta). É membro fundador e cocoordenador da Rede Luso-Brasileira de pesquisa em Artes e Intervenções Urbanas (RAIU), coordenador adjunto do GT de Cultura Visual da SOPCOM (Associação Portuguesa de Ciências da Comunicação) e coeditor da revista internacional Cadernos de Arte \& Antropologia. É autor das obras "Introdução à cultura Visual. Abordagens teóricas e metodológicas" (Mundos Sociais, 2013), "Porque pintamos a cidade? Uma abordagem etnográfica ao graffiti urbano" (Fim de Século, 2010) e co-organizador dos livros "Uma cidade de Imagens" (Mundos Sociais, 2011), "Popular \& Visual Culture: Design, Circulation and Consumption" (Cambridge Scholars Publishing, 2014) e "Transglobal Sounds. Music, indentity and migrant descendants" (Bloomsbury Academic Publishing, 2016).

\rmocampos@yahoo.com.br 
Cornelia Eckert é graduada em História (1981, UFRGS, Brasil), Mestre em Antropologia Social (1985, UFRGS, Brasil) e Doutora em Antropologia Social (1992, Université Paris V, Sorbonne, França), com pós-doutorado no Laboratoire d'Anthropologie Visuelle e Sonore du Monde Contemporaine na Université Paris VII (2001), no Instituto de Estudos Latino-Americanos da Freie Universität Berlin Rüdesheimer (2013) e na University of Georgia (2018). Professora do Departamento de Antropologia e do Programa de Pós-Graduação em Antropologia Social, IFCH, UFRGS. Pesquisadora CNPq, Bolsa Produtividade 1B. Coordena com Ana Luiza Carvalho da Rocha, o Banco Projeto Imagem e Efeitos Visuais (Laboratório de Antropologia Social) PPGAS, IFCH, UFRGS, em Porto Alegre, Brasil. Coordena o Núcleo de Antropologia Visual (NAVISUAL) (Laboratório de Antropologia Social) PPGAS, IFCH, UFRGS, em Porto Alegre, Brasil.

\section{\ chicaeckert@gmail.com}

Andrea Barbosa é Professora de Antropologia no Departamento de Ciências Sociais da UNIFESP-Universidade Federal de São Paulo. É mestre em Antropologia Social (Museu Nacional/UFRJ, 1994) e doutora em Ciência Social (Antropologia Social/ Universidade de São Paulo, 2003). Coordena desde 2007 o VISURB- Grupo de Pesquisas Visuais e urbanas da UNIFESP. É pesquisadora do GRAVI-Grupo de antropologia visual da USP desde 1996 onde participou de três projetos temáticos. É autora dos livros "São Paulo Cidade Azul" (Alameda/FAPESP, 2012) e "Antropologia e Imagem" (Zahar, 2006). É organizadora e autora dos Livros "A Experiência da Imagem na Etnografia" (Terceiro Nome, 2016), "Ciências Sociais em Diálogo" (Ed. UNIFESP, 2014), "Imagem-conhecimento" (Papirus, 2009), "Escrituras da Imagem" (EDUSP, 2004). É autora dos filmes Pimentas nos Olhos (2015), Míriam Moreira Leite: Caminhos da Memória (2007), No canto dos Olhos (2006), Em(si) Mesma (2006, Prêmio Associação dos Documentaristas do Rio de Janeiro), entre outros. 